\title{
Monitores Automáticos de Pressão Arterial. Avaliação de Três Modelos em Voluntárias *
}

\section{Automatic Blood Pressure Monitors. Evaluation of Three Models in Volunteers}

Luiz Eduardo Imbelloni, TSA ${ }^{1}$; Lúcia Beato, TSA ${ }^{2}$; Ana Paula Tolentino ${ }^{3}$; Dulcimar Donizete de Souza ${ }^{4}$; José Antônio Cordeiro ${ }^{5}$

\section{RESUMO}

Imbelloni LE, Beato L, Tolentino AP, Souza DD, Cordeiro JA Monitores Automáticos de Pressão Arterial. Avaliação de Três Modelos em Voluntárias

JUSTIFICATIVA E OBJETIVOS: Desde 1903 a monitorização da pressão arterial tem sido realizada por método não invasivo, com esfigmomanômetro ou aparelhos automáticos não invasivos. Um dos problemas da medida da pressão arterial não invasiva é considerar a variação da pressão arterial com o método utilizado. O método oscilométrico de medida da pressão arterial avalia a pressão arterial durante a deflação do manguito. Dificuldades da medida da pressão arterial pelo método oscilométrico podem acontecer por: tamanho inadequado do manguito, incorreta aplicação do manguito, faltas não detectadas pelo manguito e conectores, movimento do braço, estado de choque e compressão vascular proximal ao manguito. Este estudo avaliou a confiabilidade dos aparelhos nas medidas da pressão arterial pelo método não invasivo em cinco medidas com três aparelhos diferentes.

MÉTODO: Foi avaliada a pressão arterial em 60 voluntárias com idades entre 20 e 40 anos no período das 7 às 11 horas da manhã, na posição sentada de um dia normal de trabalho. A medida da pressão arterial constou de cinco aferições com intervalo de 2 minutos. Foram estudados três aparelhos automáticos de medida da pressão arterial. Nenhuma paciente era obesa, hipertensa ou sofria de doença cardíaca ou disritmias cardíacas. A medida indireta da pressão arterial era feita de acordo com as instruções dos fabricantes.

RESULTADOS: Não há diferença entre os três grupos estudados em relação aos parâmetros demográficos. A variação média intrapessoal na PAS, de uma medida para outra, foi de até $6,7 \mathrm{mmHg}$, na PAM de até 4,9 $\mathrm{mmHg}$ e na PAD de até $3,3 \mathrm{mmHg}$, todas com intervalo de confiança de $95 \%$. A diferença máxima entre as medidas na mesma voluntária foi de $49 \mathrm{mmHg}$ na PAS, $46 \mathrm{mmHg}$ na PAM e $28 \mathrm{mmHg}$ na PAD.

\footnotetext{
${ }^{*}$ Recebido do (Received from) Instituto de Anestesia Regional, Faculdade de Medicina de São José do Rio Preto, SP (FAMERP)

1. Diretor do Instituto de Anestesia Regional, Hospital de Base da FAMERP; Anestesiologista da Clínica São Bernardo, Rio de Janeiro, RJ

2. Anestesiologista da Clínica São Bernardo, Rio de Janeiro, $R J$

3. Anestesiologista do Hospital de Base da FAMERP

4. Chefe do Departamento de Especialidades Cirúrgicas da FAMERP

5. Coordenador de Ensino na FAMERP
}

Apresentado (Submitted) em 21 de março de 2003

Aceito (Accepted) para publicação em 30 de maio de 2003

Endereço para correspondência (Correspondence to)

Dr. Luiz Eduardo Imbelloni

Av. Epitácio Pessoa, 2356/203 Lagoa

22471-000 Rio de Janeiro, RJ

E-mail: imbelloni@openlink.combr

(C) Sociedade Brasileira de Anestesiologia, 2004
CONCLUSÕES: Este trabalho mostrou que há variação significativa entre as medidas das PAS, PAM e PAD e que a $P A D$ é o parâmetro mais fidedigno para verificar alterações da pressão arterial em voluntárias.

Unitermos: MONITORIZAÇÃO: pressão arterial; TÉCNICA DE MEDIÇÃO: pressão arterial não invasiva, oscilometria

\section{SUMMARY}

Imbelloni LE, Beato L, Tolentino AP, Souza DD, Cordeiro JA Automatic Blood Pressure Monitors. Evaluation of Three Models in Volunteers

BACKGROUND AND OBJECTIVES: Since 1903, blood pressure has been noninvasively monitored (NIBP), either with manual sphygmomanometer or automated noninvasive devices. One NIBP measurement problem is the considerable variance in blood pressure data, both within and between available techniques. The oscillometric method for NIBP monitoring evaluates blood pressure during cuff deflation. Difficulties in blood pressure measurement by oscillometry may arise from: inadequate cuff size, inadequate cuff application, undetected fails in cuff, hoses, or connectors, arm movement, shock and vascular compression proximal to the cuff. This study aimed at evaluating the reliability of three noninvasive blood pressure monitoring devices during five measurements.

METHODS: Blood pressure of 60 healthy female volunteers aged 20 to 40 years was evaluated from 7 am to $11 \mathrm{am}$, in the sitting position during a normal workday. Five measures were taken with each device at 2-minute intervals. Three automatic blood pressure monitors were studied. No patient was obese, hypertensive or suffering from cardiac disease and cardiac arrhythmia. Indirect measurements were made according to manufacturers' instructions.

RESULTS: There were no differences in demographics among the three studied groups. Mean intrapersonal variation from one measurement to the other was up to $6.7 \mathrm{mmHg}$ for systolic blood pressure (SBP), $4.9 \mathrm{mmHg}$ for mean blood pressure $(M B P)$ and $3.3 \mathrm{mmHg}$ for diastolic blood pressure (DBP) with 95\% confidence interval. The highest difference between measures in the same volunteer was $49 \mathrm{mmHg}$ for SBP, $46 \mathrm{mmHg}$ for MBP and $28 \mathrm{mmHg}$ for $D B P$.

CONCLUSIONS; This study has shown significant variations in $S B P, M B P$ and $D B P$ and that $S B P$ is the most reliable parameter to check blood pressure changes in volunteers.

Key Words: MEASUREMENT TECHNIQUES:non-invasive blood pressure, oscilometry; MONITORING: blood pressure

\section{INTRODUÇÃO}

A primeira referência da diferença da pressão sangüínea lentre os vasos deve-se ao pintor renascentista Giovanni Di Paolo, ao representar a decapitação de São João Batista, que mostrou vasos jorrando sangue (artérias) e outros gotejando sangue (veias) ${ }^{1}$. Em 1896, Riva-Rocci descre- 
veu em duas publicações sucessivas um novo esfigmomanômetro de coluna de mercúrio, que pesquisava a variação da pressão arterial sangüínea ${ }^{2,3}$.

É importante a uniformização dos procedimentos técnicos em estudos epidemiológicos, envolvendo diferentes observadores, cujos erros sistemáticos podem comprometer os resultados, e mais ainda, erros introduzidos pelo observador, principalmente em relação à pressão arterial que possui uma variabilidade intrínseca. A determinação rotineira da pressão arterial ainda é feita de maneira não padronizada, quase sempre sem observação das recomendações básicas para evitar erros nas aferições ${ }^{4,5}$. Vários fatores relacionados aos equipamentos, ao observador, ao ambiente, à paciente e à técnica propriamente dita podem interferir na precisão da medida da pressão arterial. Os critérios a serem seguidos na medida da pressão arterial são claramente explicitados em nosso meio ${ }^{6}$. A rígida observância do correto procedimento de medida da pressão arterial deve ser seguida não só na prática clínica, mas principalmente no campo da investigação clínica, nos experimentos que usam a medida da pressão arterial em seu método. Ao se avaliar a medida da pressão arterial por meio da produção científica, verificou-se que, de uma listagem de 19 itens a serem seguidos, apenas $20 \%$ atenderam a 12 deles ${ }^{5}$. Em outro estudo, analisando 223 artigos, pertencentes a 18 periódicos nacionais relacionados à prática clínica médica, no período de 1989 a 1994, resultaram em que metade dos artigos não especificou o tipo de esfigmomanômetro utilizado ${ }^{7}$. Nos trabalhos em que os tipos de aparelhos foram citados, $29 \%$ foram de coluna de mercúrio, $16 \%$ aneróides e $4 \%$ eletrônicos ${ }^{7}$. Além disso, a verificação da calibração não foi citada em $82 \%$, somente $18 \%$ expressaram preocupação em verificar a acurácia do sistema e $25 \%$ dos artigos citaram a posição da medida ${ }^{7}$. De 1999 a 2002 foram publicados 274 trabalhos na Revista Brasileira de Anestesiologia, dos quais 118 estudaram a pressão arterial e em nenhum desses foi citado o apareIho empregado nem houve qualquer relato sobre sua acurácia. Desses 274 trabalhos, em 15 foram estudadas as alterações hemodinâmicas na raquianestesia. Estudando a eficácia da efedrina na prevenção da hipotensão arterial em cesariana, também os aparelhos utilizados não foram citados ${ }^{8,9}$. A identificação de pacientes com maior risco de desenvolver hipotensão arterial durante raquianestesia já foi objeto de estudo do qual foram identificados como fatores de previsão a idade acima de 45 anos, o sexo feminino e o nível de bloqueio sensitivo acima de $\mathrm{T}_{7}{ }^{10}$.

O presente estudo avaliou três aparelhos de medidas de pressão arterial pelo método não invasivo, comumente utilizados em salas de cirurgia e aparelhos de anestesia, em um projeto de investigação em voluntárias de 20 a 40 anos, sem história de hipertensão arterial ou uso de qualquer medicação, com objetivo de verificar a confiabilidade das medidas intermitentes da pressão arterial.

\section{MÉTODO}

Foram avaliadas 60 voluntárias com idades entre 20 e 40 anos (auxiliares de enfermagem, enfermeiras, instrumenta- doras e médicas) que trabalhavam em centros cirúrgicos da Clínica São Bernardo e do Hospital Barra D'Or no Rio de Janeiro e do Hospital de Base de São José do Rio Preto, no período das 7 às 11 horas da manhã de um dia normal de trabaIho. Todas as voluntárias apresentavam pressão arterial dentro da normalidade e não faziam uso de nenhuma medicação anti-hipertensiva, não eram obesas nem tinham história de disritmias. As voluntárias que apresentaram hipertensão arterial durante a gestação foram automaticamente excluídas do estudo. A medida da pressão arterial constou de cinco aferições com intervalo de dois minutos, efetuadas em salas dos centros cirúrgicos e de acordo com recomendações do Consenso Brasileiro para tratamento da hipertensão arterial ${ }^{6}$. Todas as medidas das voluntárias foram realizadas na posição sentada e o membro superior direito mantido na altura do coração. Foi perguntado se o intervalo utilizado de dois em dois minutos trazia algum desconforto.

Para o estudo foram utilizados três aparelhos das seguintes marcas: Anamed-Vital Line AM 78100B, Hewlett-Packard modelo Viridia 24 C e Dixtal DX 2010. A medida indireta da pressão arterial era feita de acordo com as instruções dos fabricantes.

Os efeitos do tempo, aparelho (profissão) e a interação entre tempo e aparelho (tempo e profissão) foram analisados pelo método análise de medidas repetidas. Idade, peso, variação da $1^{\text {a }}$ à $5^{\mathrm{a}}$ medidas e estatura foram comparadas segundo aparelho (profissão) por Análise de Variância. As variações gerais entre as medidas foram comparadas pelo teste $t \mathrm{~Pa}-$ reado e os intervalos de confiança para as variações médias foram determinados utilizando a distribuição normal, uma vez que todas passaram no teste de normalidade.

\section{RESULTADOS}

Não há evidência de diferença entres as médias em relação aos grupos por idade, peso e altura, nem segundo o aparelho ou profissão.

\section{Comparação de Aparelho e Tempo}

Pressão arterial sistólica (PAS): há evidência de efeito de tempo ( $p=0,017)$ com a média intrapessoal caindo com o tempo; não há evidência de efeito de aparelho, nem de interação entre tempo e aparelho $(p=0,36$ e $p=0,84$, respectivamente) (Figura 1 ).

Pressão arterial diastólica (PAD): há evidência de efeito de aparelho (HP dando valores médios menores que as outras duas marcas) ( $p=0,000)$; não há evidência de efeito de tempo, nem de interação entre tempo e aparelho $(p=0,12$ e $p=$ 0,68 , respectivamente) (Figura 2).

Pressão arterial média (PAM): há evidência de efeito de aparelho $(p=0,000), H P$ dando valores médios menores que as outras duas marcas, e há evidência de efeito de tempo ( $p=$ 0,041 ) com a média intrapessoal diminuindo com o tempo, não há evidência de interação entre tempo e aparelho ( $p=$ 0,82 ) (Figura 3). 


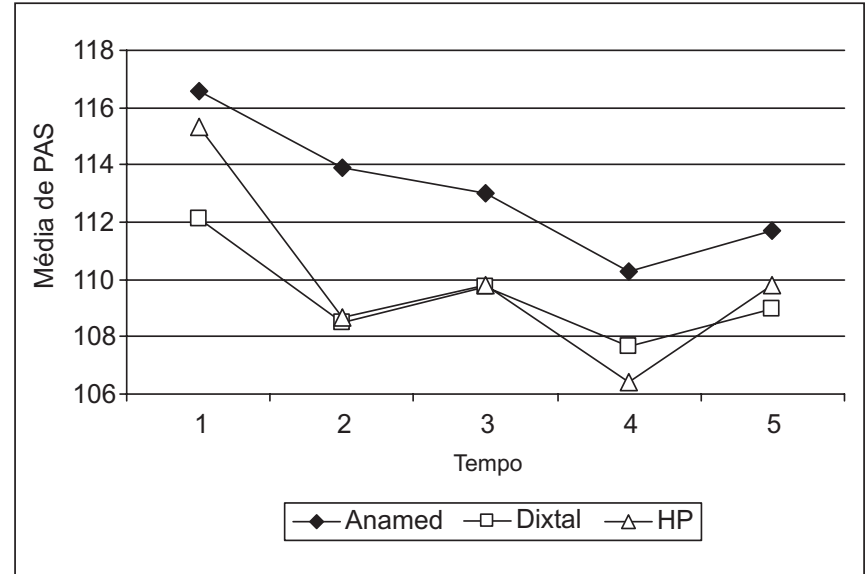

Figura 1 - Pressão Arterial Sistólica em Função do Tempo, Segundo o Aparelho

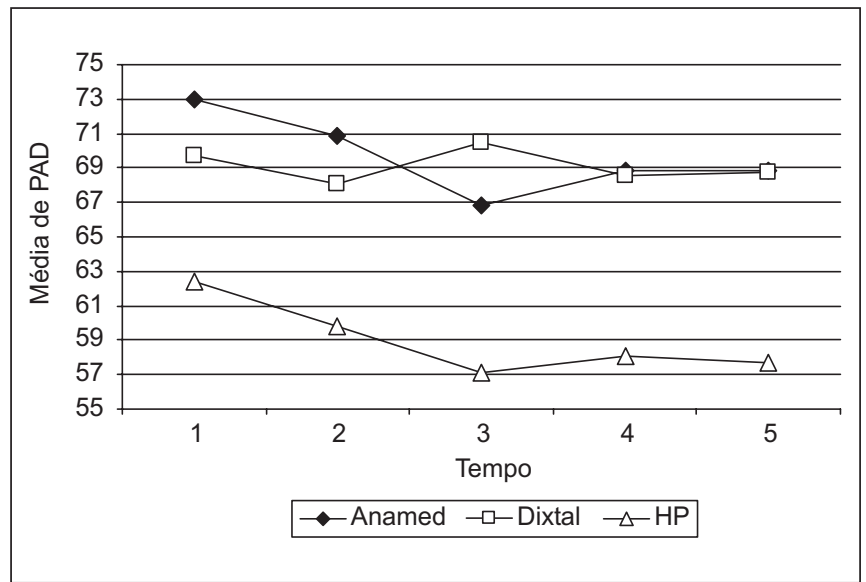

Figura 2 - Pressão Arterial Diastólica em Função do Tempo, Segundo o Aparelho

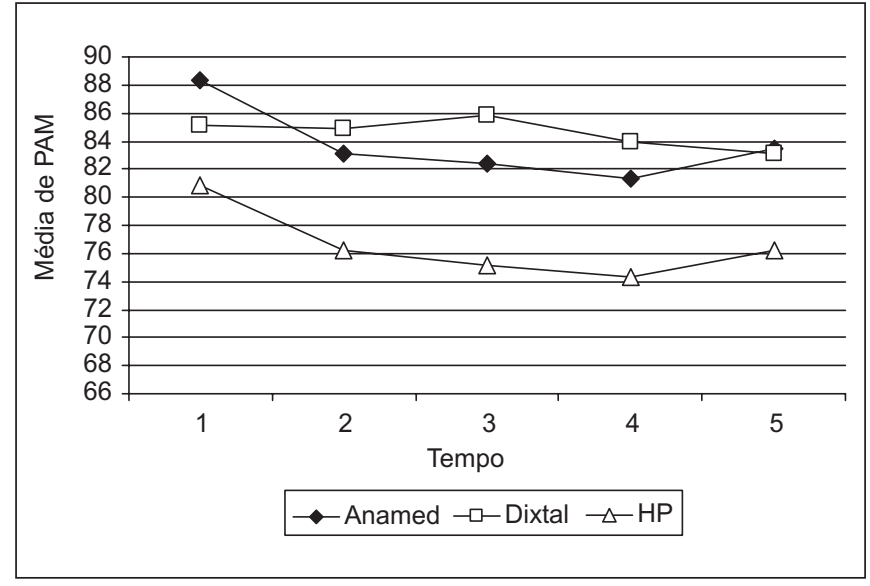

Figura 3 - Pressão Arterial Média em Função do Tempo, Segundo o Aparelho

Revista Brasileira de Anestesiologia

Vol. 54, № 1, Janeiro - Fevereiro, 2004

\section{Comparação de Profissão e Tempo}

Pressão arterial sistólica (PAS): há evidência de efeito de profissão ( $p=0,000)$, com instrumentadora dando valores médios menores que as outras, e há evidência de efeito de tempo $(p=0,014)$ com a média intrapessoal diminuindo com o tempo; não há evidência de interação entre tempo e profissão $(p=0,47)$ (Figura 4).

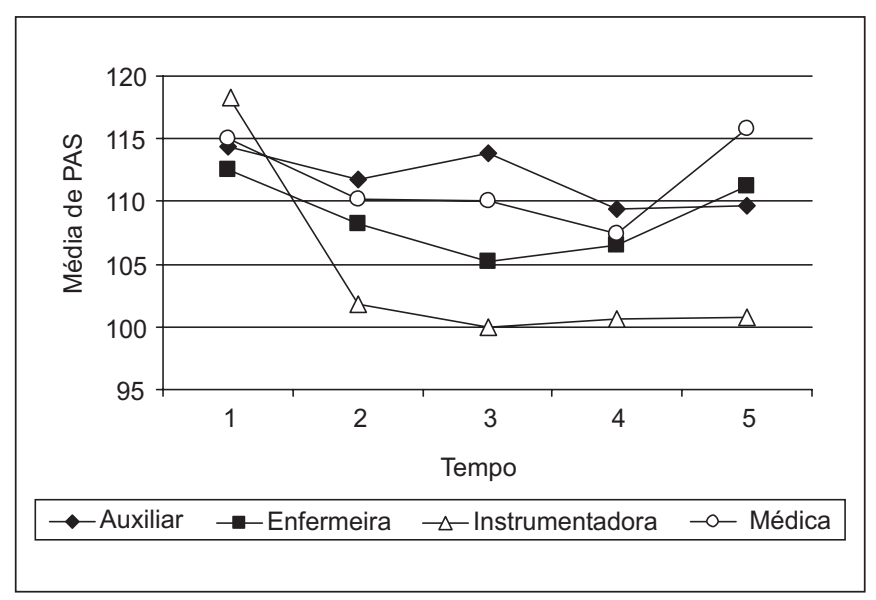

Figura 4 - Pressão Arterial Sistólica em Função do Tempo, Segundo a Profissão

Pressão arterial diastólica (PAD): há evidência de efeito de profissão $(p=0,000)$ com instrumentadora dando valores médios menores que auxiliar e médica; não há evidência de efeito de tempo $(p=0,14)$ nem de interação entre tempo e profissão $(p=0,98)$ (Figura 5$)$.

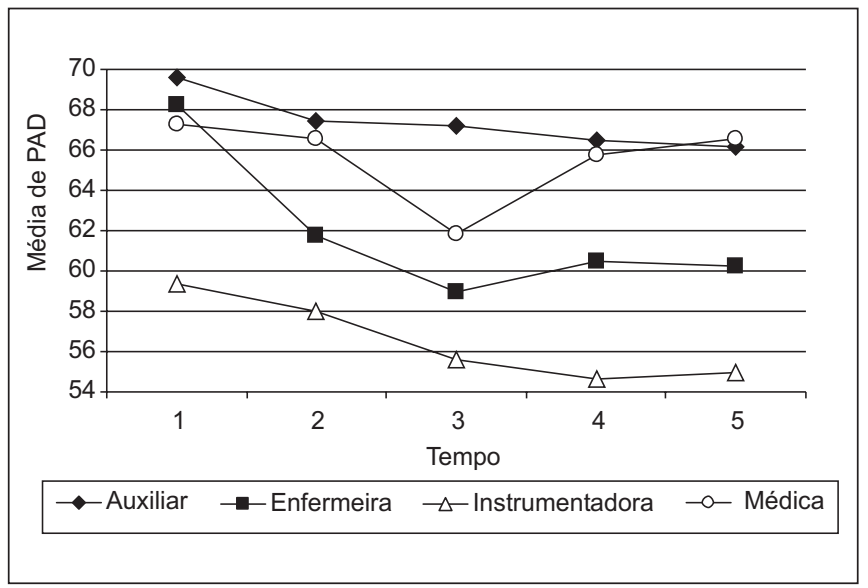

Figura 5 - Pressão Arterial Diastólica em Função do Tempo, Segundo a Profissão

Pressão arterial média (PAM): há evidência de efeito de profissão $(p=0,000)$ com instrumentadora dando valores médios menores que auxiliar e médica e há evidência de efeito de tempo ( $p=0,041)$ com média intrapessoal diminuindo, não há evidência de interação entre tempo e profissão $(p=0,71)$ (Figura 6). 


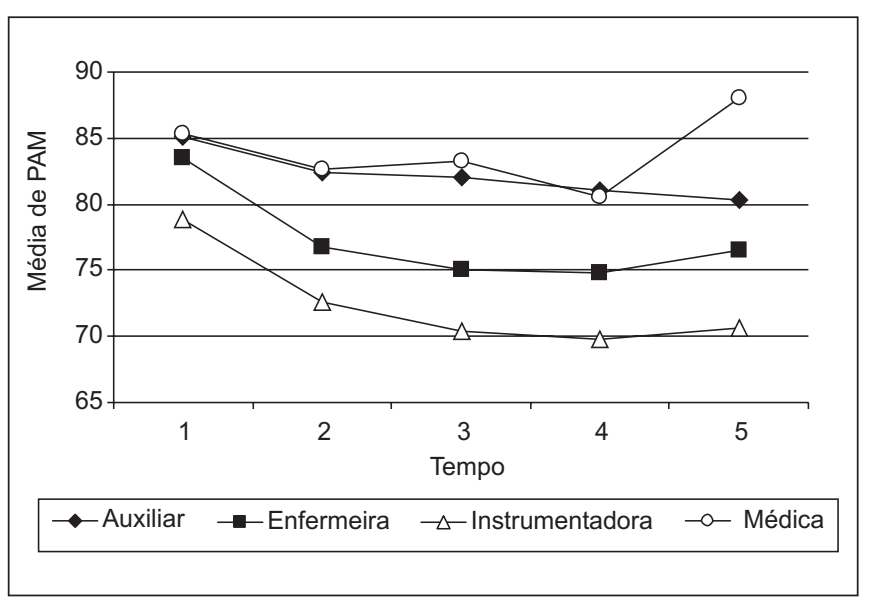

Figura 6 - Pressão Arterial Média em Função do Tempo, Segundo a Profissão

\section{Comparação da Variação Intrapessoal ao Longo do Tempo}

A variação intrapessoal média (para baixo) na PAS, de uma medida para outra, foi de até $6,7 \mathrm{mmHg}$, com intervalo de confiança $95 \%$ de 4,3 a $9 \mathrm{mmHg}$, de até $4,9 \mathrm{mmHg}$ na PAM, com intervalo de confiança $95 \%$ de 3,1 a $6,7 \mathrm{mmHg}$, e na PAD de até $3,3 \mathrm{mmHg}$ com intervalo de confiança $95 \%$ de 1,7 a 4,8 $\mathrm{mmHg}$, sendo significantes todas as diminuições médias entre a $1^{\mathrm{a}}$ e a $5^{\mathrm{a}}$ medidas. Essas variações não dependem do aparelho (valores-p > 0,25 para todas as pressões), mas pode depender da profissão. A diminuição média entre a $1^{\mathrm{a}} \mathrm{e}$ a $5^{\mathrm{a}}$ medidas, em PAS, foi maior $(17 \mathrm{mmHg})$ em instrumentadora que nas outras categorias, estas não se diferenciando entre si $(p=0,000)$, em PAM foi menor em médicas (aumento de $2,6 \mathrm{mmHg}$ ) que nas outras categorias, com estas tendo quedas médias não diferentes entre si $(p=0,009)$. Com PAD a diminuição da $1^{\mathrm{a}}$ para a $5^{\mathrm{a}}$ medidas não teve efeito significante de profissão $(p=0,46)$.

A diferença máxima entre as medidas numa mesma voluntária foi de $49 \mathrm{mmHg}$ na PAS, $46 \mathrm{mmHg}$ na PAM e $28 \mathrm{mmHg}$ na PAD. Todas estas variações máximas ocorreram do $6^{\circ}$ para o $8^{\circ}$ minutos. Em nenhuma voluntária obteve-se o mesmo valor durante as cinco medidas, mostrando uma variação intrínseca pessoal.

Averificação de dois em dois minutos cursou com uma ampla variabilidade entre as medidas e $25 \%$ queixaram do garroteamento.

\section{DISCUSSÃO}

Este trabalho mostrou que existe variação entre as medidas da PAS, da PAM e da PAD em voluntárias saudáveis e sem história de hipertensão arterial. A média máxima da variação intrapessoal de uma medida para outra foi de até $6,7 \mathrm{mmHg}$ na PAS, de até $4,9 \mathrm{mmHg}$ na PAM e de até $3,3 \mathrm{mmHg}$ na PAD, mostrando que a PAD é a que apresentou menor variação entre as medidas. A diferença máxima entre as medidas intrapessoais foi de $49 \mathrm{mmHg}$ na PAS, de $46 \mathrm{mmHg}$ na PAM e de
$28 \mathrm{mmHg}$ na PAD, todas entre a penúltima e última medida. Desta forma, as quedas das PAS e a PAM não são os melhores parâmetros para avaliação da pressão arterial em voluntárias, assim como há uma grande variação entre as primeiras e as últimas medidas, levando ao erro intrapessoal.

A técnica da medida indireta da pressão arterial usando a oclusão arterial e um estetoscópio de acordo com Riva-Roc$\mathrm{Ci}^{2,3}$ e Korotkoff ${ }^{11}$ permanece até nossos dias. Embora a medida indireta da pressão arterial com este método seja fácil de ensinar e rápida de realizar, uma medida automática é desejável, principalmente na sala de cirurgia e unidade de terapia intensiva, quando verificações repetidas são necessárias. Uma das grandes vantagens para a adoção de aparelhos automáticos é a possibilidade de afastar a influência do observador na medida da pressão arterial. A determinação da pressão arterial é necessária para o manuseio do paciente anestesiado. Métodos não invasivos são rotineiramente empregados pelo baixo risco e por sua simplicidade. Ointeresse das técnicas não invasivas que avaliam a pressão arterial média tem aumentado, porque esta pressão é freqüentemente avaliada com técnicas invasivas. O desenvolvimento dos aparelhos que avaliam a pressão arterial por método oscilométrico começou em 1973 e colocados na sala de operações em $1976^{12}$. Desde sua introdução, diversos estudos descreveram a acurácia dos aparelhos que avaliam a pressão arterial média por método indireto em recém-nascidos, crianças e adultos ${ }^{13-17}$. Embora haja exceção, muitos desses estudos mostraram que a acurácia desses aparelhos é menor de $5 \mathrm{mmHg}$ de erro médio com desvio padrão de menos de $8 \mathrm{mmHg}$ quando comparados com colocação de cateter na artéria central. Foi observada uma variabilidade intrapessoal de uma medida para outra de 4,3 a $9 \mathrm{mmHg}$ na PAS, de 3,1 a $6,7 \mathrm{mmHg}$ na PAM e de $1,7 \mathrm{a} 4,8 \mathrm{mmHg}$ na PAD, independentemente do aparelho utilizado, mostrando que a PAS e a PAM não são um bom parâmetro para avaliação de pequenas diminuições em voluntárias em dia normal de trabalho. Em uma mesma voluntária, a diminuição da PAS de uma medida para outra chegou a $49 \mathrm{mmHg}$, da PAM a 46 $\mathrm{mmHg}$ e da PAD a $28 \mathrm{mmHg}$, revelando uma grande variabilidade intrapessoal durante a aferição da pressão arterial.

Durante a anestesia, várias decisões de tratamento da pressão arterial são baseadas nas medidas das pressões arteriais sistólica e diastólica. Em pacientes graves, valores da pressão arterial média são obtidos através de punção da artéria radial. Já foi sugerido que monitores não invasivos podem perfeitamente substituir os métodos invasivos de medidas da pressão arterial ${ }^{18}$. Foi demonstrado que existe uma variação de $14 \mathrm{mmHg}$ com um intervalo de confiança de $95 \%$ para a medida da PAM em 19 pacientes ${ }^{13}$. Neste trabalho, encontramos uma variação de $4,9 \mathrm{mmHg}$ na PAM com intervalo de confiança de $95 \%$. O valor limite de $80 \mathrm{mmHg}$ no monitor oscilométrico mostrou uma tendência de superestimar a avaliação da pressão arterial acima deste limite e de subestimar a avaliação abaixo deste limite ${ }^{19}$. E com hipotensão arterial deliberada foi mostrado que o monitor oscilométrico su- 
perestima a pressão arterial ${ }^{19}$. Os coeficientes de correlação entre o método invasivo e não invasivo apresentam uma boa, mas ampla variabilidade entre os indivíduos ${ }^{20}$. Este estudo mostrou uma ampla variabilidade entre os indivíduos. As possíveis variações entre os sujeitos podem ser explicadas pela largura do manguito utilizado na avaliação pelo método oscilométrico, pois largura equivalente a um terço ou metade da circunferência do braço não produz um erro maior do que $\pm 5 \mathrm{mmHg}^{21}$. Este fator sozinho não explica as variações entre os indivíduos. Os manguitos utilizados neste estudo estavam dentro da padronização dos fabricantes para pacientes adultos, e, portanto, não contribuíram para a ampla variação nas medidas.

A hipotensão arterial e a bradicardia são os efeitos colaterais mais temidos da raquianestesia ${ }^{22,23}$, principalmente em obstetrícia, pois o tratamento pronto e eficaz é considerado fundamental para prevenir o sofrimento fetal. O critério de hipotensão arterial usado na maioria dos trabalhos de raquianestesia na Revista Brasileira de Anestesiologia de 1999 a 2003 é a diminuição de $20 \%{ }^{8-10,24-26}$ a $30 \%{ }^{27-35}$ da PAS de controle ou PAS menor que $90 \mathrm{mmHg}{ }^{10}$. Tradicionalmente, o critério de hipotensão arterial em obstetrícia é definido como sendo diminuição da PAS igual ou superior a $20 \%$ em relação ao controle, ou ainda, PAS inferior a $100 \mathrm{mmHg}$, devendo ser aferida a cada três minutos ${ }^{36}$. Atualmente, a pressão arterial é monitorizada em intervalos mais freqüentes (a cada minuto até o nascimento) e a terapêutica com vasopressor é iniciada precocemente, sendo que quedas de $10 \%$ da pressão arterial de controle são corrigidas ${ }^{36}$. Aparelhos de medidas de pressão arterial não invasiva levam em torno de 45 segundos para a insuflação, deflação e leitura. Com o método oscilométrico (em contraste com o método manual auscultatório) há pequeno efeito na acurácia da medida quando não se permite baixar a engorgitação venosa. Deve-se permitir o fluxo venoso, pelo menos um pouco, ao final de cada medida, para evitar desconforto e possível edema ou petéquia. No modo automático normal, quando o tempo de medida é regulado para um minuto, o menor intervalo entre as medidas é de 25 segundos, aproximadamente ${ }^{25}$. No modo imediato, o tempo entre as medidas é de apenas uns poucos segundos, mas, porque estas determinações imediatas levam apenas uns 15 segundos, normalmente não há desconforto nem grande engorgitamento venoso na parte distal do membro. Contudo, para prevenir qualquer possibilidade de complicações com o engorgitamento venoso, o modo imediato não deve ser usado com freqüência maior que a necessária ${ }^{37}$. Neste estudo, $25 \%$ das voluntárias queixaram-se do garroteamento durante a verificação de dois em dois minutos.

O critério de se esperar uma redução de $20 \%$ da pressão arterial sistólica para se administrar doses terapêuticas de efedrina em raquianestesia para cesariana, freqüentemente recomendada na literatura e empregada na clínica, é igualmente inadequado ${ }^{8}$. Assim, alguns autores propõem ${ }^{38}$ e outros concordam ${ }^{8}$ que a melhor forma de tratar a hipotensão arterial é o uso terapêutico de vasopressor em bolus a qualquer diminuição da pressão arterial. O uso de doses em bolus $15 \mathrm{mg}$ ou maiores do que $2 \mathrm{mg}$. $\mathrm{min}^{-1}$ determinou alta incidên- cia de hipertensão arterial e o uso profilático da efedrina desenvolveu hipertensão arterial em $15 \%$ a $17,5 \%{ }^{9}$ ou mesmo hipertensão inaceitável ${ }^{8}$. Doses iguais ou maiores que 15 mg em bolus, ou iguais ou maiores de $4 \mathrm{mg} \cdot \mathrm{min}^{-1}$, em infusão prolongada, não devem ser utilizadas, uma vez que podem induzir à alta incidência de hipertensão arterial materna ou prejudicar o bem estar fetal ${ }^{8}$. A avaliação da pressão arterial em voluntárias de dois em dois minutos mostrou que devemos ser mais prudentes no tratamento de qualquer diminuição da pressão arterial sistólica, pois pode resultar em hipertensão arterial, tão indesejável quanto a hipotensão. Em nenhuma voluntária obteve-se o mesmo valor durante as cinco medidas, mostrando uma variação intrínseca pessoal. A pressão arterial média é menos afetada pelas mudanças do tônus vascular que a pressão diastólica, porque é obtida quando as oscilações atingem sua maior amplitude durante a deflação do manguito ${ }^{25}$. Esta propriedade permite que a pressão arterial média seja usada com confiança, mesmo em casos de hipotensão arterial com vasoconstrição e diminuição da pressão de pulso ${ }^{37}$. Diferentemente, este trabalho mostrou que a PAD é que sofre a menor variação em voluntárias não submetidas ao estresse cirúrgico.

Ao avaliarmos os três aparelhos em voluntárias com idades entre 20 e 40 anos, percebemos variações intrapessoais, com diferenças médias significantes entre elas, de até 6,7 $\mathrm{mmHg}$ na PAS, de até $4,9 \mathrm{mmHg}$ na PAM e de até $3,3 \mathrm{mmHg}$ na PAD. Desta forma, tratar qualquer diminuição da pressão arterial sem levar em conta o erro médio do aparelho e a variação intrapessoal pode resultar em hipertensão arterial em torno de $17 \%{ }^{9}$ ou mesmo hipertensão arterial inaceitável ${ }^{8}$ durante raquianestesia para cesariana. A avaliação de dois em dois minutos foi desconfortável para $25 \%$ das pacientes. Este trabalho mostrou que a PAD é o parâmetro mais fidedigno para verificar alterações da pressão arterial em voluntárias. O tratamento da pressão arterial durante raquianestesia baseado em qualquer queda da PAS deve ser reavaliado.

\section{Automatic Blood Pressure Monitors. Evaluation of Three Models in Volunteers}

Luiz Eduardo Imbelloni, TSA, M.D., Lúcia Beato, TSA, M.D., Ana Paula Tolentino, M.D., Dulcimar Donizete de Souza, M.D., José Antonio Cordeiro, M.D.

\section{INTRODUCTION}

The difference in blood pressure among vessels was firstly referred to by the Renascent painter Giovanni Di Paolo in representing St. John Baptist's decapitation with some vessels pouring blood (arteries) and others dripping blood (veins) ${ }^{1}$. Riva-Rocci, in 1896, has described in two successive publications a new mercury column sphygmomanometer to evaluate blood pressure variations ${ }^{2,3}$. 
The standardization of technical procedures is important for epidemiologic studies involving different observers because systematic errors and especially observer-introduced errors, especially in blood pressure which has intrinsic variations, may compromise results. Routine blood pressure monitoring is still not standardized, almost always not following basic recommendations to avoid reading errors ${ }^{4,5}$. Several factors related to equipment, observer, environment, patient and the technique itself, may interfere with blood pressure measurement accuracy. Criteria to be followed during NIBP measurement are clearly stated in our country ${ }^{6}$. The strict adherence to the correct blood pressure measurement procedure should be adopted not only in the clinical practice but especially in clinical investigations and experiments including blood pressure measurement in their methods. In evaluating blood pressure measurement reported in the literature, it has been observed that, from a list of 19 items to be followed, only $20 \%$ have followed 12 of them ${ }^{5}$. In a different study analyzing 223 studies published by 18 Brazilian journals related to clinical practice in the period 1989-1994, it was observed that half the studies have not specified the type of sphygmomanometer used ${ }^{7}$. In studies where the type of device was mentioned, $29 \%$ were mercury column, $16 \%$ aneroids and $4 \%$ electronic 7 . In addition, calibration checks were not mentioned in $82 \%$, only $18 \%$ have expressed concern in checking system's accuracy and $25 \%$ of the studies have mentioned measurements position ${ }^{7}$. The Brazilian Journal of Anesthesiology has published 274 studies from 1999 to 2002, of which 118 have studied blood pressure and none has mentioned the device or its accuracy. From those 274 studies, 15 have studied hemodynamic changes during spinal anesthesia. In a study on the efficacy of ephedrine to prevent arterial hypotension during Cesarean sections, the devices were also not mentioned $^{8,9}$. The identification of patients at higher risk for arterial hypotension during spinal anesthesia has already been studied where predicting factors were considered age above 45 years, female gender and sensory block level above $\mathrm{T}_{7}{ }^{10}$. Our study has evaluated three noninvasive blood pressure measurement devices commonly used in operating rooms and anesthesia machines, in an investigation project with volunteers aged 20 to 40 years without history of arterial hypotension or use of medication, with the objective of evaluating the reliability of intermittent blood pressure measurements.

\section{METHODS}

Participated in this study 60 female volunteers, aged 20 to 40 years (assistant nurses, nurses, instrumentation technicians and physicians) working at surgical centers of Clinica São Bernardo and Hospital Barra D'Or in Rio de Janeiro, and Hospital de Base from São José do Rio Preto in the period 7 a.m. to 11 a.m. of a normal workday. All volunteers had normal blood pressures and were not under any anti-hypertensive medication, were not obese and had no history of arrhythmias. Volunteers with arterial hypertension during gestation were excluded from the study. Blood pressure was measured five times at 2-minute intervals, in surgical center rooms and according to Brazilian Consensus recommendations for arterial hypertension treatment ${ }^{6}$. All measures were obtained in the sitting position and with right arm maintained at the level of the heart. It has been asked whether the 2-minute interval adopted would bring any discomfort.

Three devices were used in the study: Anamed-Vital Line AM 78100B, Hewlett-Packard (HP) model Viridia 24 C and Dixtal DX 2010. Indirect blood pressure was obtained according to manufacturers' instructions.

Repeated measures analysis method was used to evaluate effects of time, device (profession) and interaction between time and device (time and profession). Analysis of variance was used to compare age, weight, $1^{\text {st }}$ to $5^{\text {th }}$ measures variation and height according to device (profession). Paired t test was used to compare general variations among measures and confidence intervals for mean variations were determined by normal distribution, since all were approved by the normality test.

\section{RESULTS}

There have been no differences in mean age, weight and height, or according to device of profession.

\section{Device and Time Comparison}

Systolic blood pressure (SBP): there is evidence of time effect $(p=0.017)$ with intrapersonal mean decreasing with time; there is no evidence of device effect or of interaction between time and device ( $p=0.36$ and $p=0.84$ respectively) (Figure 1).

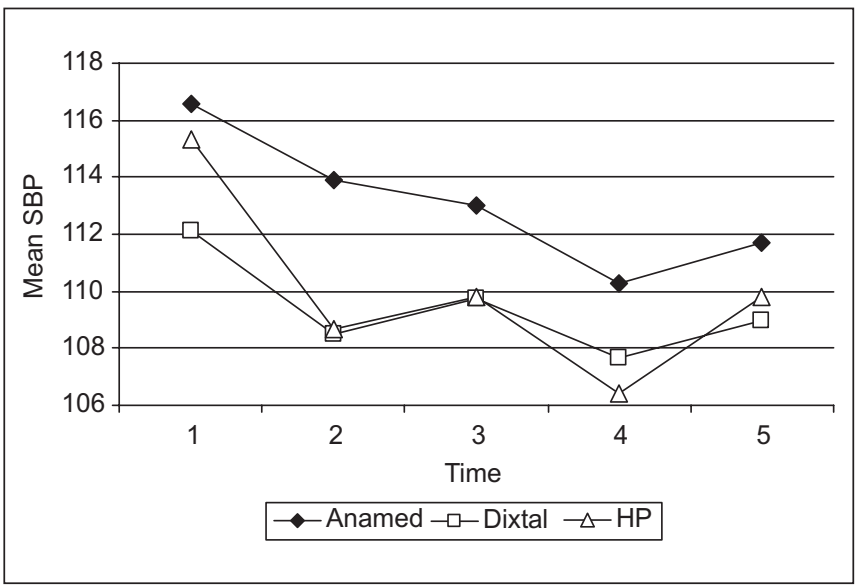

Figure 1 - Systolic Blood Pressure as a Function of Time, According to Device

Diastolic blood pressure (DBP): there is evidence of device effect (HP with mean values lower than other two brands) $(p=$ 0.000 ); there is no evidence of time effect or of interaction between time and device ( $p=0.12$ and $p=0.68$ respectively) (Figure 2). 


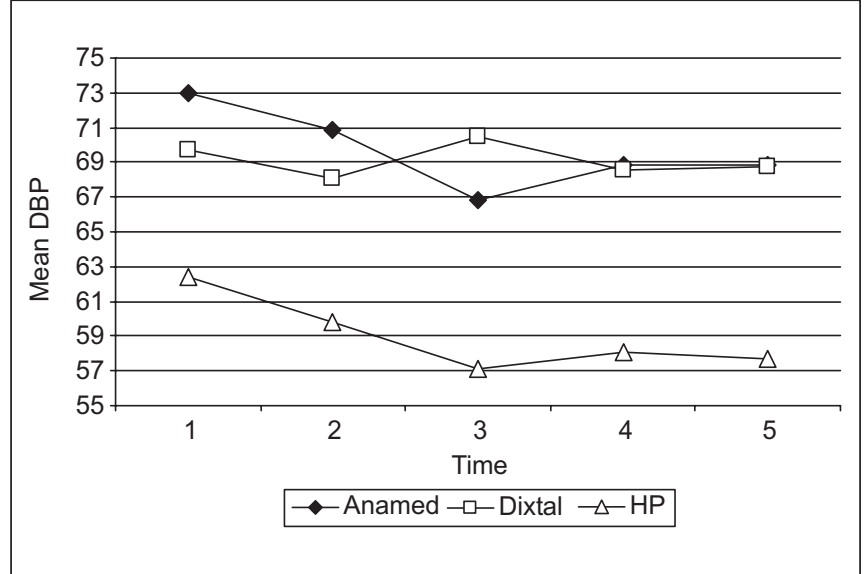

Figure 2 - Diastolic Blood Pressure as a Function of Time, According to Device

Mean blood pressure $(M B P)$ : there is evidence of device effect $(p=0.000)$ with HP showing lower mean values as compared to other brands. There is evidence of time effect $(p=$ 0.041 ) with intrapersonal mean decreasing along time. There is no evidence of interaction between time and device ( $p=$ 0.82) (Figure 3).

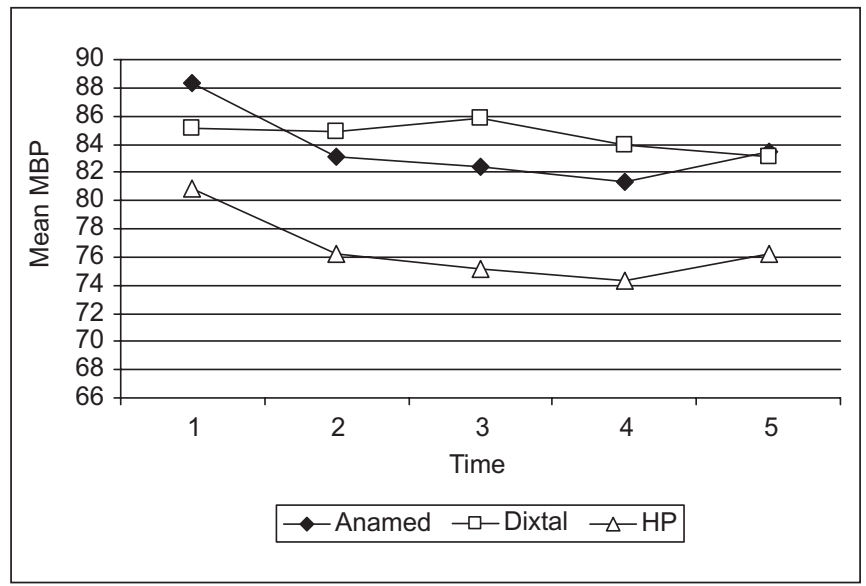

Figure 3 - Mean Blood Pressure as a Function of Time, According to Device

Profession and Time Comparison

Systolic blood pressure (SBP): there is evidence of profession effect $(p=0.000)$, with lower mean values for instrumentation technician as compared to other professions. There is evidence of time effect $(p=0.014)$ with intrapersonal mean decreasing along time. There is no evidence of interaction between time and profession $(p=0.47)$ (Figure 4).

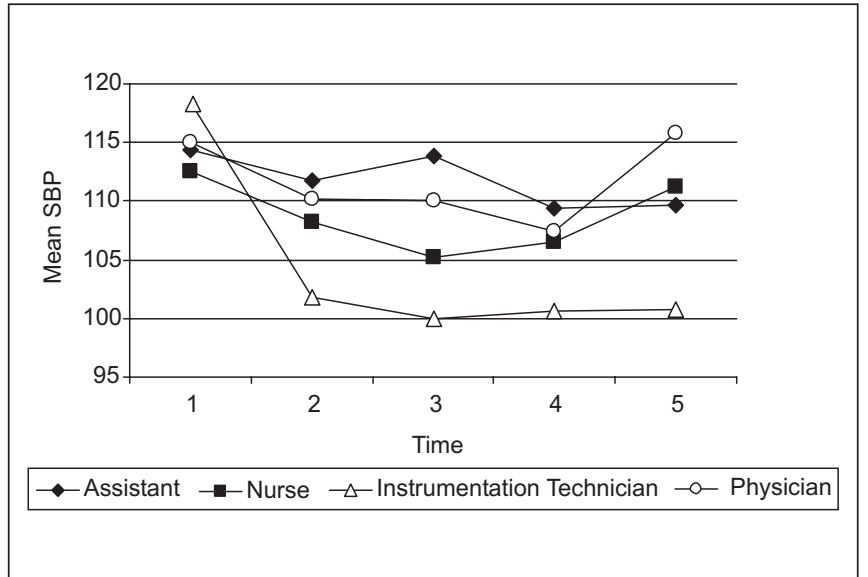

Figure 4 - Systolic Blood Pressure as a Function of Profession, According to Device

Diastolic blood pressure (DBP): there is evidence of profession effect $(p=0.000)$, with lower mean values for instrumentation technique as compared to assistant nurse and physician. There is no evidence of time effect $(p=0.14)$ or of interaction between time and profession ( $p=0.98)$ (Figure 5$)$.

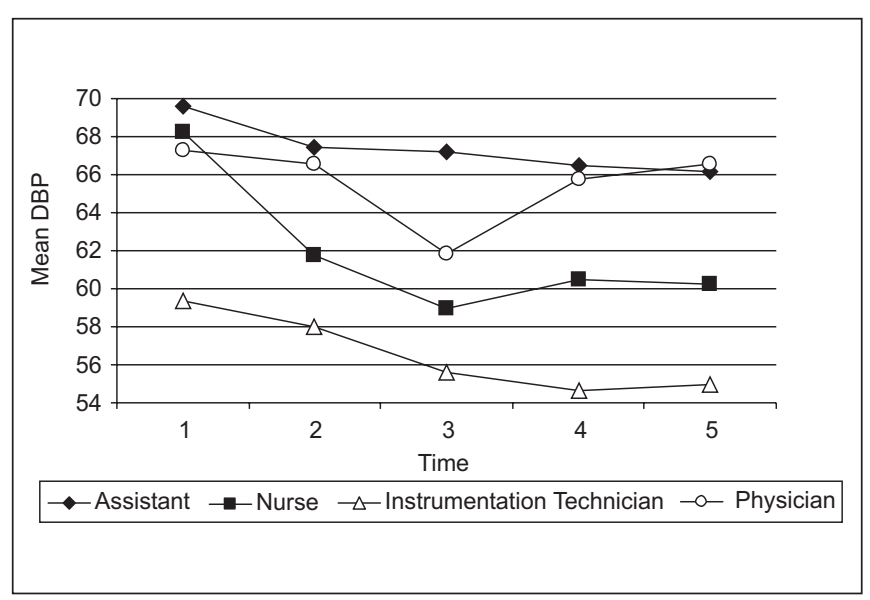

Figure 5 - Diastolic Blood Pressure as a Function of Profession, According to Device

Mean blood pressure (MBP): there is evidence of profession effect $(p=0.000$ ) with lower mean values for instrumentation technician as compared to assistant nurse and physician, and there is evidence of time effect $(p=0.041)$ with decreasing intrapersonal mean. There is no evidence of interaction between time and profession $(p=0.71)$ (Figure 6$)$. 


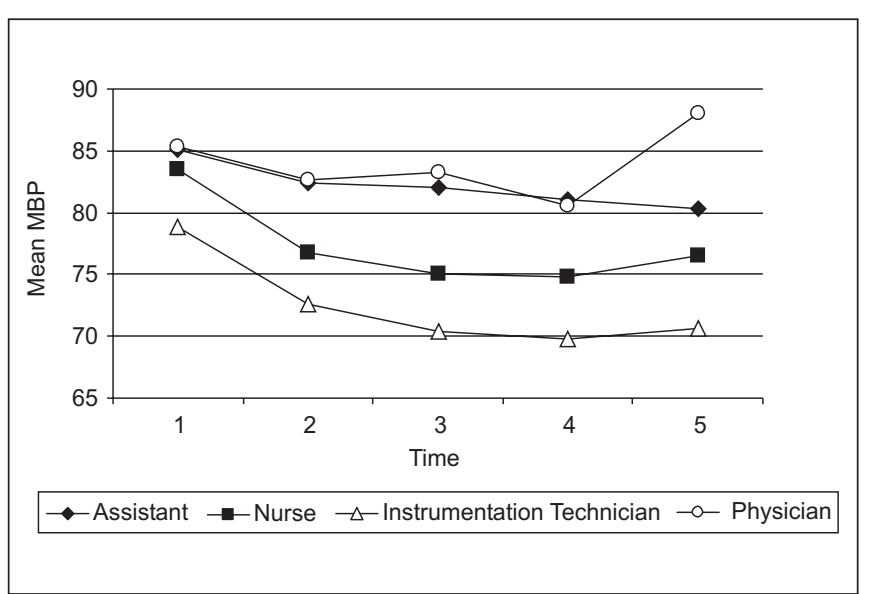

Figure 6 - Mean Blood Pressure as a Function of Profession, According to Device

Intrapersonal Variation Comparison Along Time

Mean (downwards) intrapersonal variation from one measurement to the other was: SBP up to $6.7 \mathrm{mmHg}$ (4.3 to 9 $\mathrm{mmHg}$ ) with $95 \%$ confidence interval, MBP up to $4.9 \mathrm{mmHg}$ ( 3.1 to $6.7 \mathrm{mmHg}$ ) with $95 \%$ confidence interval and MBP up to $3.3 \mathrm{mmHg}$ ( 1.7 to $4.8 \mathrm{mmHg}$ ) with $95 \%$ confidence interval being significant all mean decreases between $1^{\text {st }}$ and $5^{\text {th }}$ measurements. These variations are device-independent ( $p$ $>0.25$ for all pressures), but may be profession-dependent. Mean decrease between $1^{\text {st }}$ and $5^{\text {th }}$ SBP measurements was higher $(17 \mathrm{mmHg})$ in instrumentation technician as compared to other professions which were not different among themselves ( $p=0.000)$; MBP was lower in physicians (increase of $2.6 \mathrm{mmHg}$ ) as compared to other professions which were not different among themselves $(p=0.009)$. Mean decrease from $1^{\text {st }}$ to $5^{\text {th }}$ DBP measurements was not significantly influenced by profession $(p=0.46)$.

Maximum difference in a same volunteer was $49 \mathrm{mmHg}$ for SBP, $46 \mathrm{mmHg}$ for MBP and $28 \mathrm{mmHg}$ for DPB. All maximum variations were observed from the $6^{\text {th }}$ to the $8^{\text {th }}$ minutes. No volunteer presented the same value during the five measurements, showing an intrinsic personal variation.

The 2-minute interval checking has shown wide measurement variability and $25 \%$ of volunteers have complained about the tourniquet.

\section{DISCUSSION}

This study has shown that there are variations in SBP, MBP and DBP measurements of healthy volunteers without history of arterial hypertension. Maximum mean intrapersonal variation from one measurement to the other has been up to 6.7 $\mathrm{mmHg}$ for SBP, up to $4.9 \mathrm{mmHg}$ for MBP and up to $3.3 \mathrm{mmHg}$ for DBP, with less measurement variation for SBP. Maximum intrapersonal measurements difference has been $49 \mathrm{mmHg}$ for SBP, $46 \mathrm{mmHg}$ for MBP and $28 \mathrm{mmHg}$ for DBP, all between the $4^{\text {th }}$ and the $5^{\text {th }}$ measurement. This way, SBP and MBP decreases are not the best parameter to evaluate blood pressure in volunteers, as well as there are wide variations between $1^{\text {st }}$ and last measurements leading to intrapersonal errors.

Indirect blood pressure measurement technique using arterial occlusion and stethoscope, according to Riva-Rocci ${ }^{2,3}$ and Korotkoff ${ }^{11}$ remains to date. Although this indirect blood pressure reading is easy to teach and fast to perform, an automatic measurement is desirable, especially in the operating room and intensive care unit, when repeated measurements are needed. A major advantage of automatic devices is the possibility of ruling out the influence of the observer. Blood pressure measurement is needed to handle anesthetized patients. Noninvasive methods are routinely used due to their low risk and simplicity. The interest in noninvasive mean blood pressure techniques is increasing because this pressure is often evaluated by invasive techniques. The oscillometric method started to be developed in 1973 and was placed in the operating room in $1976{ }^{12}$. Since its introduction, several studies have described the accuracy of devices evaluating mean blood pressure by the indirect method in neonates, children and adults ${ }^{13-17}$. Although there are exceptions, several studies have shown that the accuracy of such devices is below $5 \mathrm{mmHg}$ of mean error with standard deviation of less than $8 \mathrm{mmHg}$ as compared to catheter insertion in the central artery. It has been observed an intrapersonal variability from one measurement to the other of 4.3 to $9 \mathrm{mmHg}$ for SBP, 3.1 to $6.7 \mathrm{mmHg}$ for MBP and 1.7 to $4.8 \mathrm{mmHg}$ for DBP, regardless of the device, showing that SBP and MBP are not a good parameter to evaluate minor decreases in volunteers during a normal workday. In a same volunteer, SBP decrease from one measurement to the other has reached $49 \mathrm{mmHg}$ while MBP reached $46 \mathrm{mmHg}$ and DBP $28 \mathrm{mmHg}$, showing major intrapersonal variability during blood pressure monitoring.

Several blood pressure treatment decisions during anesthesia are made based on systolic and diastolic blood pressure. In severely ill patients, mean blood pressure values are obtained through radial artery puncture. It has been suggested that noninvasive monitors may perfectly replace invasive blood pressure methods ${ }^{18}$. A $14 \mathrm{mmHg}$ MBP variation with $95 \%$ confidence interval has been shown in 19 patients ${ }^{13}$. Our study has found $4.9 \mathrm{mmHg}$ variation for MBP with $95 \%$ confidence interval. Limit $80 \mathrm{mmHg}$ value of the oscillometric monitor has shown a trend to overestimate blood pressure above this limit and underestimate the evaluation below this limit ${ }^{19}$. It has also been shown that the oscillometric monitor overestimates blood pressure in the presence of deliberate arterial hypotension ${ }^{19}$. Correlation coefficients between invasive and noninvasive methods have shown a good, however wide variability among individuals ${ }^{20}$. Possible variations among individuals may be explained by the width of the cuff used with the oscillometric method because width equivalent to one third or half the arm's circumference does not produce an error above $\pm 5 \mathrm{mmHg}^{21}$. This factor alone does not explain variations among individuals. Cuffs used in 
our study were within manufacturers' standards for adults, so have not contributed for the wide measurement variations. Arterial hypotension and bradycardia are the most feared spinal anesthesia side-effects ${ }^{22,23}$, especially in obstetrics, because prompt and effective treatment is considered critical to prevent fetal suffering. Arterial hypotension criteria used in most spinal anesthesia studies published by the Brazilian Journal of Anesthesiology from 1999 to 2003 is 20\% ${ }^{8-10,24-26}$ to $30 \%{ }^{27-35}$ decrease of control SBP or SBP below $90 \mathrm{mmHg}$ 10. Traditionally, arterial hypotension in obstetrics is defined as SBP decrease equal to or below $20 \%$ as compared to control, or even, SBP below $100 \mathrm{mmHg}$, which should be monitored at 3-minute intervals ${ }^{36}$. Currently, blood pressure is monitored in more frequent intervals (every minute till birth) and vasopressant therapy is early started to correct even $10 \%$ decrease of control pressure ${ }^{36}$. Noninvasive blood pressure devices take approximately 45 seconds for inflation, deflation and reading. The oscillometric method (as compared to manual auscultation method) has a minor effect in measurement accuracy when venous ingurgitation is not allowed to decrease. At least a little venous flow should be allowed at the end of each measurement to avoid discomfort and possible edema or petechia. In the normal automatic mode, when measurement time is set for one minute, the shortest interval between measurements is approximately 25 seconds $^{25}$. In the immediate mode, time between measurements is only a few seconds, but since these immediate measurements take approximately 15 seconds only, in general there is no discomfort or major venous ingurgitation at the distal limb. However, to prevent any possibility of complications with venous ingurgitation, the immediate mode should not be used more frequently than necessary ${ }^{37}$. In our study, $25 \%$ of volunteers have complained of tourniquet during the 2-minute interval measurements.

The criteria of waiting for $20 \%$ decrease in systolic blood pressure to administer therapeutic ephedrine doses in spinal anesthesia for Cesarean sections, often recommended in the literature and adopted in the clinic, is equally inadequate ${ }^{8}$. So, some authors have proposed ${ }^{38}$ and others agree ${ }^{8}$ that the best way to treat arterial hypotension is the therapeutic use of bolus vasopressants for any blood pressure decrease. Bolus doses of $15 \mathrm{mg}$ or higher than $2 \mathrm{mg} \cdot \mathrm{min}^{-1}$ have determined high incidence of arterial hypertension and prophylactic ephedrine has developed arterial hypertension in $15 \%$ to $17.5 \%{ }^{9}$ or even unacceptable arterial hypertension ${ }^{8}$. Bolus doses equal to or higher than $15 \mathrm{mg}$, or prolonged infusions equal to or higher than $4 \mathrm{mg} \cdot \mathrm{min}^{-1}$ should not be used since they may induce high incidence of maternal arterial hypertension or impair fetal wellbeing ${ }^{8}$. Blood pressure evaluation in volunteers at 2-minute intervals has shown that we should be more careful in treating any systolic blood pressure decrease because it may result in arterial hypertension, which is as undesirable as arterial hypotension. No volunteer had the same value in the five measurements, showing an intrinsic personal variation.

Mean blood pressure is less affected by vascular tone changes than diastolic pressure, because it is obtained when oscillations reach their highest amplitude during cuff deflation $^{25}$. This feature allows mean blood pressure to be safely used, even in cases of arterial hypotension with vasoconstriction and pulse pressure decrease ${ }^{37}$. Conversely, our study has shown that DBP presented fewer variations in volunteers not submitted to surgical stress.

In evaluating three devices in volunteers aged 20 to 40 years, we have observed intrapersonal variations with significantly different means of up to $6.7 \mathrm{mmHg}$ for SBP, $4.9 \mathrm{mmHg}$ for MBP and $3.3 \mathrm{mmHg}$ for DBP. So, treating any blood pressure decrease without taking into account mean device error and intrapersonal variation may result in approximately $17 \%$ arterial hypertension ${ }^{9}$ or even unacceptable arterial hypertension ${ }^{8}$ during spinal anesthesia for Cesarean section. The evaluation at 2-minute intervals was uncomfortable for $25 \%$ of patients. This study has shown that DBP is the best parameter to evaluate blood pressure changes in volunteers. Blood pressure treatment during spinal anesthesia based on any SBP decrease should be revaluated.

\section{REFERÊNCIAS - REFERENCES}

01. Dominguez RC, Michel A - Evolucion de la esfigmomanometria. Arch Inst Cardiol Méx, 1994;34:315-323.

02. Riva-Rocci S - Um nuovo sfigmomanometro. Gazzetta Medica di Torino, 1896;50:981-996.

03. Riva-Rocci S - Um nuovo sfigmomanometro. Gazzetta Medica di Torino, 1896;51981-1001-1017.

04. Lelhane A, O'Malley K, O'Brien ET - Reporting of blood pressure data in medical journals. Br Med J, 1980;281:1603-1604.

05. Roche V, O'Malley K, O"Brien ET - How scientific is blood pressure measurement in leading medical journal? J Hypert, 1990;8:1167-1168.

06. Consenso Brasileiro para tratamento da hipertensão arterial. Arq Bras Card, 1994;16:(Supl2):S257-S258.

07. Holanda HEM, Mion Jr D, Peirin AMG - Medida da pressão arterial. Critérios empregados em artigos científicos de periódicos brasileiros. Arq Bras Card, 1997;68:433-436.

08. Carvalho JCA, Cardoso MMSC, Capelli EL et al - Prophylactic ephedrine during cesarean delivery spinal anesthesia: dose-response study of bolus and continuous infusion administration. Rev Bras Anestesiol, 1999;49:309-314.

09. Carvalho JCA, Cardoso MMSC, Lorenz E et al - Prophylactic ephedrine during spinal anesthesia for cesarean section: bolus followed by continuous infusion in fixed doses or continuous infusion in decreasing doses. Rev Bras Anestesiol, 2000;50: 425-430.

10. Oliveira Filho GR, Garcia JHS, Goldschimidit R et al - Predictors of early hypotension during spinal anesthesia. Rev Bras Anestesiol, 2001;51:298-304.

11. Korotkoff NS - On the subject of methods of determining blood pressure. Bull Imperial Medical Academy, St. Petersburg, 1905;11:365.

12. Ramsey III M - Non-invasive automatic determination of mean arterial pressure. Med Biol Eng Comput, 1979;17:11-18.

13. Yelderman M, Ream AK - Indirect measurement of mean blood pressure in the anesthetized patient. Anesthesiology, 1979;50: 253-256.

14. Hutton P, Dye J, Prys-Roberts C - An assessment of the Dinamap 845. Anaesthesia, 1984;39:261-267. 
15. Park MK, Menard SM - Accuracy of blood pressure measurement of the Dinamap monitor in infants and children. Pediatrics, 1987;79:907-914.

16. Wareham JS, Haugh LD, Yeager SB et al - Prediction of arterial pressure in the premature neonate using the oscillometric method. Am J Dis Child, 1987;141:1108-1110.

17. Johnson CJH, Kerr JH - Automatic blood pressure monitors. A clinical evaluation of five models in adults. Anaesthesia, 1985;40:471-478

18. Moyle JTB - Non-Invasive Monitoring in Anaesthesia. IN: Kaufman L - Anaesthesia Review II. Edinburgh: Churchill Livingstone: 1984;200-213.

19. Gourdeau M, Martin R, Lamarche T et al - Oscillometry and direct blood pressure: a comparative clinical study during deliberate hypotension. Can Anaesth Soc J, 1986;33:300-307.

20. Gloyna DF, Huber P, Abston P et al - A comparison of blood pressure measurement techniques in the hypotensive patient. Anesth Analg, 1984;63:222.

21. Geddes LA, Tivey R - The importance of cuff width in measurement of blood pressure indirectly. Cardiovasc Res Cen Bul, 1976;14:69-79.

22. Carpenter RL, Caplan RA, Brown DL et al - Incidence and risk factors for side effects of spinal anesthesia. Anesthesiology, 1992;76:906-916.

23. Auroy $Y$, Naschi P, Messiah A et al - Severe complications related to regional anesthesia: Results of a prospective survey in France. Anesthesiology, 1997;87:479-486.

24. Carvalho JCA, Cardoso MMSC, Amaro AR et al - Low doses of subarachnoid morphine and fentanyl combined with rectal diclofenac for postoperative pain control after cesarean delivery. Rev Bras Anestesiol, 1999;49:103-106.

25. Delfino J, Pontes S, Gondim D et al - Isobaric $0.5 \%$ bupivacaine and $0.5 \%$ ropivacaine in spinal anesthesia for orthopedic surgery. A comparative study. Rev Bras Anestesiol, 1999;49:160-164.

26. Delfino J, Vale NB - Spinal anesthesia with $0.5 \%$ isobaric ropivacaine or levobupivacaine for lower limb surgeries. Rev Bras Anestesiol, 2001;51:91-97.

27. Imbelloni LE, Carneiro ANG - Comparison of $1.5 \%$ and $2 \%$ lidocaine with dextrose for spinal anesthesia. Rev Bras Anestesiol, 1999;49:9-13

28. Imbelloni LE, Carneiro ANG - Comparison of $2 \%$ lidocaine with or without glucose for spinal anesthesia. Rev Bras Anestesiol, 1999;49:98-102.

29. Neves JFNP, Monteiro GA, Almeida JR et al - Spinal anesthesia for cesarean section: headache evaluation with $25 \mathrm{G}$ and $27 \mathrm{G}$ Quincke and Whitacre needles. Rev Bras Anestesiol, 1999;49:173-175.

30. Alves TCA, Braz JRC, Ganem EM - Influence of pre-anesthetic medication with clonidine on the association of sufentanil and bupivacaine in spinal anesthesia. Rev Bras Anestesiol, 1999;49:320-326

31. Delfino J, Vale NB, Magalhães Filho E - Comparative study between $0.5 \%$ and $0.75 \%$ isobaric ropivacaine in spinal anesthesia for orthopedic surgery. Rev Bras Anestesiol, 2000;50: 207-211.

32. Imbelloni LE, Beato L - Comparative study between $0.5 \%$ isobaric bupivacaine and $0.5 \%$ isobaric mixture of $75 \% \mathrm{~S}(-)$ bupivacaine and $25 \% \mathrm{R}(+)$ bupivacaine in spinal anesthesia for orthopedic surgery. Rev Bras Anestesiol, 2001;51:369-376.

33. Imbelloni LE - Spinal anesthesia with $2 \%$ plain lidocaine for short orthopedic surgery. Study in 250 patients. Rev Bras Anestesiol, 2002;52:24-33.
34. Imbelloni LE, Vieira EM, Beato L et al - Spinal anesthesia for outpatient pediatric surgery in $1-5$ years old children with $0.5 \%$ isobaric enantiomeric mixture of bupivacaine (S75:R25). Rev Bras Anestesiol, 2002;52:286-293.

35. Imbelloni LE, Vieira EM, Beato L et al - Isobaric $0.5 \%$ bupivacaine for spinal anesthesia in pediatric outpatient surgery of 6 to 12 year old children. A prospective study. Rev Bras Anestesiol, 2002;52:402-409.

36. Cardoso MMSC - Hipotensão arterial materna em raquianestesia para cesárea. Cedar, 2002;VI:20:4-6.

37. Ramsey III M - Blood pressure monitoring: automated oscillometric devices. J Clin Monitoring, 1991;7:56-67.

38. Datta S, Alper MH, Ostheimer GW et al - Method of ephedrine administration and nausea and hypotension during spinal anesthesia during cesarean section. Anesthesiology, 1982;56: 68-70.

\section{RESUMEN}

Imbelloni LE, Beato L, Tolentino AP, Souza DD, Cordeiro JA Monitores Automáticos de Presión Arterial. Evaluación de Tres Modelos en Voluntarias

JUSTIFICATIVA Y OBJETIVOS: Desde 1903 la monitorización de la presión arterial ha sido realizada por método no invasor, con esfigmomanómetro o aparatos automáticos no invasores. Uno de los problemas de la medida de la presión arterial no invasora es considerar la variación de la presión arterial con el método utilizado. El método oscilométrico de medida de la presión arterial evalúa la presión arterial durante la deflación del manguito. Dificultades de la medida de la presión arterial por el método oscilométrico pueden acontecer por: tamaño inadecuado del manguito, incorrecta aplicación del manguito, faltas no detectadas por el manguito y conectores, movimiento del brazo, estado de choque y compresión vascular proximal al manguito. Este estudio evaluó la confianza de los aparatos en las medidas de la presión arterial por el método no invasor en cinco medidas con tres aparatos diferentes.

MÉTODO: Fue evaluada la presión arterial en 60 voluntarias con edades entre 20 y 40 años en el período de las 7 a las 11 horas de la mañana, en la posición sentada de un día normal de trabajo. La medida de la presión arterial constató de cinco afericiones con intervalo de 2 minutos. Fueron estudiados tres aparatos automáticos de medida de la presión arterial. Ninguna paciente era obesa, hipertensa o sofría de enfermedad cardíaca o disritmias cardíacas. La medida indirecta de la presión arterial era hecha de acuerdo con las instrucciones de los fabricantes.

RESULTADOS: No hay diferencia entre los tres grupos estudiados en relación a los parámetros demográficos. La variación media intrapersonal en la PAS, de una medida para otra, fue de hasta $6,7 \mathrm{mmHg}$, en la PAM de hasta $4,9 \mathrm{mmHg}$ y en la PAD de hasta $3,3 \mathrm{mmHg}$, todas con intervalo de confianza $95 \%$. La diferencia máxima entre las medidas en la misma voluntaria fue de $49 \mathrm{mmHg}$ en la PAS, $46 \mathrm{mmHg}$ en la PAM y 28 $\mathrm{mmHg}$ en la $P A D$

CONCLUSIONES: Este trabajo mostró que hay variación significativa entre as medidas de las PAS, PAM y PAD y que la $P A D$ es el parámetro más fidedigno para verificar alteraciones de la presión arterial en voluntarias. 